Article

\title{
Some New Results on F-Contractions in 0-Complete Partial Metric Spaces and 0-Complete Metric-Like Spaces
}

\author{
Stojan Radenović $^{1}$ (D), Nikola Mirkov ${ }^{2, *(D)}$ and Ljiljana R. Paunović ${ }^{3}$ (D) \\ 1 Faculty of Mechanical Engineering, University of Belgrade, Kraljice Marije 16, 11120 Belgrade, Serbia; \\ radens@beotel.net \\ 2 "Vinča" Institute of Nuclear Sciences-National Institute of the Republic of Serbia, University of Belgrade, \\ Mike Petrovića Alasa 12-14, 11351 Belgrade, Serbia \\ 3 Teacher Education Faculty, University in Priština-Kosovska Mitrovica, Nemanjina b.b, \\ 38218 Leposavić, Serbia; lijijana.paunovic@pr.ac.rs \\ * Correspondence: nmirkov@vin.bg.ac.rs
}

Citation: Radenović, S.; Mirkov, N.; Paunović, L.R. Some New Results on F-Contractions in 0-Complete Partial Metric Spaces and 0-Complete Metric-Like Spaces. Fractal Fract. 2021, 5, 34. https://doi.org/ 10.3390 / fractalfract5020034

Academic Editors: Ricardo Almeida and Maria Rosaria Lancia

Received: 5 February 2021

Accepted: 8 April 2021

Published: 19 April 2021

Publisher's Note: MDPI stays neutral with regard to jurisdictional claims in published maps and institutional affiliations.

Copyright: (C) 2021 by the authors Licensee MDPI, Basel, Switzerland. This article is an open access article distributed under the terms and conditions of the Creative Commons Attribution (CC BY) license (https:/ / creativecommons.org/licenses/by/ $4.0 /$ )
Abstract: Within this manuscript we generalize the two recently obtained results of O. Popescu and G. Stan, regarding the F-contractions in complete, ordinary metric space to 0-complete partial metric space and 0-complete metric-like space. As Popescu and Stan we use less conditions than D. Wardovski did in his paper from 2012, and we introduce, with the help of one of our lemmas, a new method of proving the results in fixed point theory. Requiring that the function $F$ only be strictly increasing, we obtain for consequence new families of contractive conditions that cannot be found in the existing literature. Note that our results generalize and complement many well-known results in the fixed point theory. Also, at the end of the paper, we have stated an application of our theoretical results for solving fractional differential equations.

Keywords: metric-like spaces; partial metric spaces; 0-cauchy sequence; F-contractions; strictly increasing

MSC: 47H10; 54H25

\section{Introduction and Preliminaries}

Our exposition starts by looking back on some basic concepts, notations, and established results for metric, metric-like and partial metric spaces.

Metric spaces were introduced in 1906 by Maurice Fréchet in his seminal work [1] as follows:

Definition 1. Let $X$ be a nonempty set. A mapping $d^{m}: X^{2} \rightarrow[0,+\infty)$ is said to be a metric on $X$ if for all $\bar{x}, \bar{y}, \bar{z} \in X$ the following three conditions hold:

$\left(d^{m} 1\right) d^{m}(\bar{x}, \bar{y})=0$ if and only if $\bar{x}=\bar{y}$;

$\left(d^{m} 2\right) d^{m}(\bar{x}, \bar{y})=d^{m}(\bar{y}, \bar{x})$;

$\left(d^{m} 3\right) d^{m}(\bar{x}, \bar{z}) \leq d^{m}(\bar{x}, \bar{y})+d^{m}(\bar{y}, \bar{z})$.

If $d^{m}$ is a metric on $X$, then the pair $\left(X, d^{m}\right)$ is said to be a metric space. The theory of metric spaces contains several branches of mathematical analysis: real analysis, complex analysis and multidimensional analysis (for more details, see [2]).

Partial metric spaces were introduced in 1994 by Matthews [3] as follows.

Definition 2. Let $X$ be a nonempty set. A mapping $d^{p m}: X^{2} \rightarrow[0,+\infty)$ is said to be a partial metric on $X$ if for all $\bar{x}, \bar{y}, \bar{z} \in X$ the following four conditions hold:

$\left(d^{p m} 1\right) \bar{x}=\bar{y}$ if and only if $d^{p m}(\bar{x}, \bar{x})=d^{p m}(\bar{x}, \bar{y})=d^{p m}(\bar{y}, \bar{y})$;

$\left(d^{p m} 2\right) d^{p m}(\bar{x}, \bar{x}) \leq d^{p m}(\bar{x}, \bar{y})$ 


$$
\begin{aligned}
& \left(d^{p m} 3\right) d^{p m}(\bar{x}, \bar{y})=d^{p m}(\bar{y}, \bar{x}) \\
& \left(d^{p m} 4\right) d^{p m}(\bar{x}, \bar{z}) \leq d^{p m}(\bar{x}, \bar{y})+d^{p m}(\bar{y}, \bar{z})-d^{p m}(\bar{y}, \bar{y}) .
\end{aligned}
$$

Then, the pair $\left(X, d^{p m}\right)$ is called a partial metric space. It can be checked that every metric space is also a partial metric space. The opposite is not true. In that spirit, let $X=[0,+\infty)$ and partial metric be defined as $d^{p m}(x, y)=\max \{x, y\}$. Under these circumstances $\left(X, d^{p m}\right)$ constitutes a partial metric space but it does not constitute a metric space, since $d^{p m}(1,1)=1>0$. To acquire more details on this, we point to following works [4-9]:

Metric-like spaces were introduced in 2012 by A. Amini Harandi [10] in following way:

Definition 3. Let $X$ be a nonempty set. A mapping $d^{m l}: X^{2} \rightarrow[0,+\infty)$ is said to be a metric-like on $X$ if for $\bar{x}, \bar{y}, \bar{z} \in X$ the following three conditions hold:

$$
\begin{aligned}
& \left(d^{m l} 1\right) d^{m l}(\bar{x}, \bar{y})=0 \text { yields } \bar{x}=\bar{y} ; \\
& \left(d^{m l} 2\right) d^{m l}(\bar{x}, \bar{y})=d^{m l}(\bar{y}, \bar{x}) ; \\
& \left(d^{m l} 3\right) d^{m l}(\bar{x}, \bar{z}) \leq d^{m l}(\bar{x}, \bar{y})+d^{m l}(\bar{y}, \bar{z}) .
\end{aligned}
$$

The pair $\left(X, d^{m l}\right)$ is called a metric-like space or dislocated metric space in some papers. A metric-like mapping $d^{m l}$ on $X$ satisfies all the conditions of a metric except that $d^{m l}(x, x)$ may be positive for some $x \in X$. Below we assemble a short list of representative examples of partial metric and metric-like spaces:

1. $\left(\mathbb{R}, d^{p m}\right)$, where $d^{p m}(\bar{x}, \bar{y})=\max \{|\bar{x}|,|\bar{y}|\}$ for all $\bar{x}, \bar{y} \in \mathbb{R}$.

It can be seen that $\left(\mathbb{R}, d^{p m}\right)$ is a partial metric space, and a metric-like space, but it is not a metric space, due to the fact that $d^{p m}(|-2|,|-2|)=2>0$.

2. $\left([0,+\infty), d^{m l}\right)$, where $d^{m l}(\bar{x}, \bar{y})=\bar{x}+\bar{y}$ for all $\bar{x}, \bar{y} \in[0,+\infty)$.

It can be checked that $\left([0,+\infty), d^{m l}\right)$ is a metric-like space where $d^{m l}(\bar{x}, \bar{x})>0$ for each $\bar{x}>0$. Since $d^{m l}(2,2)=2+2=4>3=2+1=d^{m l}(2,1)$, it follows that $d^{m l}(\bar{x}, \bar{x}) \leq d^{m l}(\bar{x}, \bar{y})$ does not hold. Hence, $\left([0,+\infty), d^{m l}\right)$ is not a partial metric space.

3. $\left(X, d^{m l}\right)$, where $X=\{0,1,2\}$ and $d^{m l}(0,0)=d^{m l}(1,1)=0, d^{m l}(2,2)=\frac{5}{2}$, $d^{m l}(0,2)=d^{m l}(2,0)=2, d^{m l}(1,2)=d^{m l}(2,1)=3, d^{m l}(0,1)=d^{m l}(1,0)=\frac{3}{2}$.

Also, it can be seen that $\left(X, d^{m l}\right)$ is a metric-like (that is a dislocated metric) space with $d^{m l}(2,2)>0$. This means that $\left(X, d^{m l}\right)$ is not a standard metric space. However, $\left(X, d^{m l}\right)$ also is not a partial metric space, because $d^{m l}(2,2) \not d^{m l}(2,0)$.

4. $\left(X, d^{m l}\right)$, where $X=C([0,1], \mathbb{R})$ is the set of real continuous functions on $[0,1]$ and $d^{m l}(u, v)=\sup _{t \in[0,1]}(|u(t)|+|v(t)|)$ for all $u, v \in C([0,1], \mathbb{R})$.

This is an example of metric-like space that is not a partial metric space. Indeed, for $u(t)=2 t$, we obtain $d^{m l}(u, u)=\sup _{t \in[0,1]}(2 t+2 t)=4>0$. Putting $v(t) \equiv 0$ for all $t \in[0,1]$, we obtain that $d^{m l}(u, u)=4 \not d^{m l}(u, v)=d^{m l}(u, 0)=2$.

We note here that some of the metric-like spaces exemplified in the former list do not represent partial metric spaces. We also note that a partial metric space also represents a metric-like space but the opposite is not true. In the sequel, we will give the definitions related to sequences in metric-like spaces, on their convergence and Cauchyness (for more details, see [7,9-13]).

Definition 4. Let $\left\{x_{n}\right\}$ be a sequence in a metric-like space $\left(X, d^{m l}\right)$.

(i) $\left\{x_{n}\right\}$ is said to converge to $\bar{x} \in X$ if $\lim _{n \rightarrow+\infty} d^{m l}\left(x_{n}, \bar{x}\right)=d^{m l}(\bar{x}, \bar{x})$;

(ii) $\left\{x_{n}\right\}$ is said to be $d^{m l}$-Cauchy in $\left(X, d^{m l}\right)$ if $\lim _{n, p \rightarrow+\infty} d^{m l}\left(x_{n}, x_{p}\right)$ exists and is finite; 
(iii) A metric-like space $\left(X, d^{m l}\right)$ is $d^{m l}$-complete if for every $d^{m l}$-Cauchy sequence $\left\{x_{n}\right\}$ in $X$ there exists an $x \in X$ such that

$$
\lim _{n, p \rightarrow+\infty} d^{m l}\left(x_{n}, x_{p}\right)=d^{m l}(x, x)=\lim _{n \rightarrow+\infty} d^{m l}\left(x_{n}, x\right) .
$$

Interested readers can find more details on metric-like and partial metric spaces in following selected references (e.g., [3,6,7,9-12]). Further investigation on generalization of metric spaces to other classes of generalized metric spaces and on definition of contractive mappings can be found in variety of papers [2,13-20]:

Remark 1. Following remark is valid for the metric-like spaces, and also in the case of partial metric spaces. Notably, as examples in Remark 1.4 (1) and (2) in [6] illustrate, a sequence doesn't need to have a unique limit and a convergent sequence doesn't need to be a $d^{m l}$-Cauchy sequence. On the other hand, if the sequence $\left\{x_{n}\right\}$ is $d^{m l}$-Cauchy sequence in a way that $\lim _{n, p \rightarrow+\infty} d^{m l}\left(x_{n}, x_{p}\right)=0$ holds in $d^{m l}$-complete metric-like space $\left(X, d^{m l}\right)$, then such a sequence has a unique limit. Demonstrably, in such a case if $x_{n} \rightarrow x$ as $n \rightarrow+\infty$, we get that $d^{m l}(x, x)=0$ (from condition (iii) of Definition 4). Now, if $x_{n} \rightarrow x, x_{n} \rightarrow y$ and $x \neq y$, we get

$$
d^{m l}(x, y) \leq d^{m l}\left(x, x_{n}\right)+d^{m l}\left(x_{n}, y\right) \rightarrow d^{m l}(x, x)+d^{m l}(y, y)=0+0=0 .
$$

Under the condition $\left(d^{m l} 1\right)$ from Definition 3, what follows is that $x=y$, which forms a contradiction.

Otherwise, the sequence $\left\{x_{n}\right\}_{n \in \mathbb{N}}$ in metric-like space $\left(X, d^{m l}\right)$ is called $0-d^{m l}$-Cauchy if $\lim _{n, p \rightarrow+\infty} d^{m l}\left(x_{n}, x_{p}\right)=0$. In this case the metric-like space $\left(X, d^{m l}\right)$ is $0-d^{m l}$-complete if in it each $0-d^{m l}$-Cauchy sequence is convergent. Since every $0-d^{m l}$-Cauchy sequence is $d^{m l}$-Cauchy it yields that $d^{m p}$-complete partial metric space is $0-d^{m l}$-complete.

In the continuation of our exposition we present some statements valid for metric-like spaces, for which the proofs are immediate.

Proposition 1. Let $\left(X, d^{m l}\right)$ be a metric-like space and $\left\{x_{n}\right\}$ be a sequence in it. Then we have the following:

(i) If $\left\{x_{n}\right\}$ converges to $\bar{x} \in X$ as $n \rightarrow+\infty$ and if $d^{m l}(\bar{x}, \bar{x})=0$, then for all $\bar{y} \in X$ it follows that $d^{m l}\left(x_{n}, \bar{y}\right) \rightarrow d^{m l}(\bar{x}, \bar{y})$;

(ii) If $d^{m l}(\bar{x}, \bar{y})=0$ then $d^{m l}(\bar{x}, \bar{x})=d^{m l}(\bar{y}, \bar{y})=0$;

(iii) If $\lim _{n \rightarrow+\infty} d^{m l}\left(x_{n}, x_{n+1}\right)=0$ then $\lim _{n \rightarrow+\infty} d^{m l}\left(x_{n}, x_{n}\right)=$ $\lim _{n \rightarrow+\infty} d^{m l}\left(x_{n+1}, x_{n+1}\right)=0$;

(iv) If $\bar{x} \neq \bar{y}$ then $d^{m l}(\bar{x}, \bar{y})>0$;

(v) $d^{m l}(\bar{x}, \bar{x}) \leq \frac{2}{n} \sum_{i=1}^{n} d^{m l}\left(\bar{x}, x_{i}\right)$ holds for all $\bar{x}, x_{i} \in X$, where $1 \leq i \leq n$;

(vi) Let $\lim _{n \rightarrow+\infty} d^{m l}\left(x_{n}, x_{n+1}\right)=0$. If $\lim _{n, p \rightarrow+\infty} d^{m l}\left(x_{n}, x_{p}\right) \neq 0$, then there exists $\varepsilon>0$ and sequences $\{n(k)\}$ and $\{p(k)\}$ such that $n(k)>p(k)>k$, and the following sequences tend to $\varepsilon^{+}$when $k \rightarrow+\infty$ :

$$
d^{m l}\left(x_{n(k)}, x_{p(k)}\right), d^{m l}\left(x_{n(k)+1}, x_{p(k)}\right), d^{m l}\left(x_{n(k)}, x_{p(k)-1}\right), d^{m l}\left(x_{n(k)+1}, x_{p(k)-1}\right) .
$$

If the condition (vi) is satisfied then the sequences $d^{m l}\left(x_{n(k)+q}, x_{p(k)}\right)$ and $d^{m l}\left(x_{n(k)+q}, x_{p(k)+1}\right)$ also converge to $\varepsilon^{+}$when $k \rightarrow+\infty$, where $q \in \mathbb{N}$. For more details on (i)-(vi) the reader can see $[7,13]$. 
(vii) If $\left\{x_{n}\right\}_{n \in \mathbb{N} \cup\{0\}}$ is a Picard sequence in a metric-like space $\left(X, d^{m l}\right)$ induced by a mapping $T: X \rightarrow X$ and if $d^{m l}\left(x_{n}, x_{n+1}\right)<d^{m l}\left(x_{n-1}, x_{n}\right)$ for all $n \in \mathbb{N}$ then $x_{n} \neq x_{m}$ whenever $n \neq m$.

In 2012, Wardowski [21] introduced a new type of mapping $T: X \rightarrow X$ named $F-$ contraction by defining a list of properties for the function $F:(0,+\infty) \rightarrow(-\infty,+\infty)$

(F1): $F$ is strictly increasing, i.e., $0<\alpha<\beta$ yields $F(\alpha)<F(\beta)$;

(F2): For each sequence $\left\{\alpha_{n}\right\}_{n \in \mathbb{N}}$ in $(0,+\infty), \lim _{n \rightarrow+\infty} \alpha_{n}=0$ if and only if $\lim _{n \rightarrow+\infty} F\left(\alpha_{n}\right)=$ $-\infty$; and

(F3): There exists $k \in(0,1)$ such that $\lim _{\alpha \rightarrow 0^{+}} \alpha^{k} F(\alpha)=0$, and proved a fixed point result as a generalization of the Banach contraction principle in different way. By $\mathcal{F}$ we denote a family of functions satisfying properties (F1-F3).

Definition 5 ([21]). Let $(X, d)$ be a metric space. A mapping $T: X \rightarrow X$ is called an F-contraction if there exists $\tau>0$ such that

$$
\tau+F(d(T x, T y)) \leq F(d(x, y))
$$

for all $x, y \in X$ with $d(T x, T y)>0$, where $F$ satisfies (F1), (F2) and (F3).

On the same line Secelean [20] changed the condition (F2) of [21] by an equivalent condition,

$\left(\mathrm{F}^{\prime}\right)^{\prime}$ : inf $F=-\infty$ or, also, by

(F2"): there exists a sequence $\left\{\alpha_{n}\right\}_{n \in \mathbb{N}}$ of positive real numbers such that $\lim _{n \rightarrow+\infty} F\left(\alpha_{n}\right)=$ $-\infty$;

and later Piri and Kumam [18] replaced condition (F3) of [21] by

$\left(\mathrm{F}^{\prime}\right)$ : $F$ is continuous on $(0,+\infty)$.

Authors in [22] take (F1) of [21] and (F3') of [18] and denote the class of functions satisfying (F1) and (F3') by $\triangle_{F}$. For more new results in this subject see [16,23-27].

\section{Main Results}

After giving the overview of the results related to metric, partial metric and metric-like spaces, as well as recollecting the notion of an F-contraction, the properties of function family involved with it, as well as some recent variations of the required set of function properties, we move to the main goal of the paper, which is an attempt to generalize, complement, unify, enrich and extend all the results recently obtained in [24]. Namely, firstly in [28] authors introduced and proved the following:

Definition 6 ([28]). Let $(X, d)$ be a metric space. A mapping $T: X \rightarrow X$ is called an F-contraction of Hardy-Rogers-type if there exist $\tau>0$ and $F \in \mathcal{F}$ such that

$$
\tau+F(d(T x, T y)) \leq F(A(x, y))
$$

holds for any $x, y \in X$ with $d(T x, T y)>0$, where $A(x, y)=\alpha \cdot d(x, y)+\beta \cdot d(x, T x)+$ $\gamma \cdot d(y, T y)+\delta \cdot d(x, T y)+L \cdot d(y, T x), \alpha, \beta, \gamma, \delta, L$ are non-negative numbers, $\gamma \neq 1$ and $\alpha+\beta+\gamma+2 \delta=1$.

Theorem 1 ([28]). Consider $(X, d)$ to be a complete metric space and let $T$ be a mapping of $X$ into itself. If one assumes that $T$ is an F-contraction of Hardy-Rogers- type, with $\gamma \neq 1$, then the mapping $T$ has a fixed point. Further, if $\alpha+\beta+L \leq 1$ holds, then the fixed point of the mapping $T$ is unique.

After that, authors in [24] proved the next proper generalization of results from [28]: 
Theorem 2 ([24]). Let $T$ be a self-mapping of a complete metric space X. Suppose that there exists $\tau>0$ such that for all $x, y \in X, d(T x, T y)>0$ yields

$$
\tau+F(d(T x, T y)) \leq F(A(x, y)),
$$

where $F:(0,+\infty) \rightarrow(-\infty,+\infty)$ is a strictly increasing mapping, $A(x, y)=\alpha \cdot d(x, y)+$ $\beta \cdot d(x, T x)+\gamma \cdot d(y, T y)+\delta \cdot d(x, T y)+L \cdot d(y, T x), \alpha, \beta, \gamma, \delta, L$ are non-negative numbers, $\delta<\frac{1}{2}, \gamma<1, \alpha+\beta+\gamma+2 \delta=1,0<\alpha+\delta+L \leq 1$. Then Thas a unique fixed point $x^{*} \in X$ and for every $x \in X$ the sequence $\left\{T^{n} x\right\}_{n \in \mathbb{N}}$ converges to $x^{*}$.

Second new generalization given also in [24] shows that the monotonicity of $F$ is not a necessary condition.

Theorem 3 ([24]). Let $T$ be a self-mapping of a complete metric space $X$. If we assume that there exists $\tau>0$ such that for all $\bar{x}, \bar{y} \in X, d(T \bar{x}, T \bar{y})>0$ the following holds

$$
\tau+F(d(T \bar{x}, T \bar{y})) \leq F(d(\bar{x}, \bar{y}))
$$

where $F:(0,+\infty) \rightarrow(-\infty,+\infty)$ is a mapping satisfying conditions (F2) and $\left(F 3^{\prime \prime}\right)$, where $\left(F 3^{\prime \prime}\right): F$ is continuous on $(0, \alpha)$, with $\alpha$ being a positive real number.

Then, the mapping $T$ has a unique fixed point $x^{*} \in X$ and for every $\bar{x} \in X$ the sequence $\left\{T^{n} \bar{x}\right\}_{n \in \mathbb{N}}$ converges to $x^{*}$.

As our first result in this section are new contractive conditions that follow from the previous two theorems. They complement the ones given in $[19,29]$. Here we formulate the following:

Corollary 1. Let $(X, d)$ be a complete metric space and $T: X \rightarrow X$ be a self-mapping. Suppose that there exist $\tau_{i}>0, i=\overline{1,6}$ such that for all $x, y \in X$ the following inequalities hold:

$$
\begin{aligned}
\tau_{1}+d(T x, T y) & \leq A(x, y), \\
\tau_{2}+\exp (d(T x, T y)) & \leq \exp (A(x, y)), \\
\tau_{3}-\frac{1}{d(T x, T y)} & \leq-\frac{1}{A(x, y)}, \\
\tau_{4}-\frac{1}{d(T x, T y)}+d(T x, T y) & \leq-\frac{1}{A(x, y)}+A(x, y), \\
\tau_{5}+\frac{1}{1-\exp (d(T x, T y))} & \leq \frac{1}{1-\exp (A(x, y))}, \\
\tau_{6}+\frac{1}{\exp (-d(T x, T y))-\exp (d(T x, T y))} & \leq \frac{1}{\exp (-A(x, y))-\exp (A(x, y))},
\end{aligned}
$$

where $A(x, y)=\alpha \cdot d(x, y)+\beta \cdot d(x, T x)+\gamma \cdot d(y, T y)+\delta \cdot d(x, T y)+L \cdot d(y, T x)$, while $\alpha, \beta, \gamma, \delta$, L are non-negative numbers, $\delta<\frac{1}{2}, \gamma<1, \alpha+\beta+\gamma+2 \delta=1,0<\alpha+\delta+L \leq 1$ Then in each of these cases, there exists $x^{*} \in X$ such that $T x^{*}=x^{*}$ and for every $x \in X$ the sequence $\left\{T^{n} x\right\}_{n \in \mathbb{N} \cup\{0\}}$ converges to $x^{*}$

Proof. As each of the functions $F_{1}(\xi)=\xi, F_{2}=\exp \xi, F_{3}(\xi)=-\frac{1}{\xi}, F_{4}(\xi)=-\frac{1}{\xi}+$ $\xi, F_{5}(\xi)=\frac{1}{1-\exp \xi}, F_{6}(\xi)=\frac{1}{\exp (-\xi)-\exp \xi}$ is strictly increasing on $(0,+\infty)$, the proof immediately yields by Theorem 2 . It is clear that the proofs for (8-10) yields also by Theorem 3 .

Our second new result in this section is extending of Theorems 2 and 3 from standard metric to partial metric space. That is, we give the next: 
Theorem 4. Let $T$ be a self-mapping of a 0-complete partial metric space $\left(X, d^{p m}\right)$. Suppose there exists $\tau>0$ such that for all $x, y \in X, d^{p m}(T x, T y)>0$ yields

$$
\tau+F\left(d^{p m}(T x, T y)\right) \leq F(A(x, y)),
$$

where $F:(0,+\infty) \rightarrow(-\infty,+\infty)$ is a strictly increasing mapping, $A(x, y)=\alpha \cdot d^{p m}(x, y)+$ $\beta \cdot d^{p m}(x, T x)+\gamma \cdot d^{p m}(y, T y)+\delta \cdot d^{p m}(x, T y)+L \cdot d^{p m}(y, T x), \alpha, \beta, \gamma, \delta, L$ are non-negative numbers $\delta<\frac{1}{2}, \gamma<1, \alpha+\beta+\gamma+2 \delta+L=1,0<\alpha+\delta+L \leq 1$. Then $T$ has a unique fixed point $x^{*} \in X$ and for every $x \in X$ the sequence $\left\{T^{n} x\right\}_{n \in \mathbb{N} \cup\{0\}}$ converges to $x^{*}$.

Proof. First of all, (12) yields that

$$
d^{p m}(T x, T y)<A(x, y),
$$

whenever $d^{p m}(T x, T y)>0$. In the first step, we show that $T$ has a unique fixed point if it exists. Indeed, let $\bar{x} \neq \bar{y}$ be two distinct fixed points of $T$. From (13) follows

$$
\begin{aligned}
d^{p m}(\bar{x}, \bar{y})<\alpha \cdot d^{p m}(\bar{x}, \bar{y}) & +\beta \cdot d^{p m}(\bar{x}, \bar{x})+\gamma \cdot d^{p m}(\bar{y}, \bar{y})+\delta \cdot d^{p m}(\bar{x}, \bar{y})+L \cdot d^{p m}(\bar{y}, \bar{x}) \\
& =(\alpha+0+0+\delta+L) \cdot d^{p m}(\bar{x}, \bar{y}) .
\end{aligned}
$$

Since, $\bar{x} \neq \bar{y}$ then $d^{p m}(\bar{x}, \bar{y})>0$, therefore (14) yields $1<\alpha+0+0+\delta+L=$ $\alpha+\delta+L \leq 1$. But, this is a contradiction.

Now, we consider the Picard's sequence $x_{n}=T x_{n-1}, n \in \mathbb{N}$ induced by an arbitrary point $x_{0} \in X$. If $x_{p}=x_{p+1}$ for some $p \in \mathbb{N}$ then $x_{p}$ is a unique fixed point of $T$. Therefore, assume that $x_{n} \neq x_{n+1}$ for all $n \in \mathbb{N} \cup\{0\}$. For this case, according to (13) we get:

$$
d^{p m}\left(x_{n}, x_{n+1}\right)=d^{p m}\left(T x_{n-1}, T x_{n}\right)<A\left(x_{n-1}, x_{n}\right),
$$

where $A\left(x_{n-1}, x_{n}\right)=\alpha \cdot d^{p m}\left(x_{n-1}, x_{n}\right)+\beta \cdot d^{p m}\left(x_{n-1}, x_{n}\right)+\gamma \cdot d^{p m}\left(x_{n}, x_{n+1}\right)$

$+\delta \cdot d^{p m}\left(x_{n-1}, x_{n+1}\right)+L \cdot d^{p m}\left(x_{n}, x_{n}\right) \leq(\alpha+\beta+\delta+L) \cdot d^{p m}\left(x_{n-1}, x_{n}\right)$

$+(\gamma+\delta) \cdot d^{p m}\left(x_{n}, x_{n+1}\right)$. For the proof of the last inequality we used ( $\left.d^{p m} 2\right)$ with $x=$ $x_{n-1}, y=x_{n}$ as well as $\left(d^{p m} 4\right)$ with $x=x_{n-1}, z=x_{n+1}$. Further, (15) and the last inequality imply

$$
(1-\gamma-\delta) \cdot d^{p m}\left(x_{n}, x_{n+1}\right)<(\alpha+\beta+\delta+L) \cdot d^{p m}\left(x_{n-1}, x_{n}\right)
$$

Since, $\alpha+\beta+\gamma+2 \delta+L=1$ it follows that $1-\gamma-\delta>0$. Indeed, if $1-\gamma-\delta \leq 0$ then $1=\alpha+\beta+\gamma+2 \delta+L \geq \alpha+\beta+\gamma+\delta+L+1$, i.e., $\alpha+\beta+\gamma+\delta+L \leq 0$. This means that $\alpha=\beta=\gamma=\delta=L=0$, that is, $\gamma=1$. But this is a contradiction. Now, further (16) yields

$$
d^{p m}\left(x_{n}, x_{n+1}\right)<\frac{\alpha+\beta+\delta+L}{1-\gamma-\delta} \cdot d^{p m}\left(x_{n-1}, x_{n}\right)=d^{p m}\left(x_{n-1}, x_{n}\right),
$$

for all $n \in \mathbb{N}$. Since, the sequence $\left\{d^{p m}\left(x_{n}, x_{n+1}\right)\right\}_{n \in \mathbb{N}}$ is strictly decreasing, so there exists $\lim _{n \rightarrow+\infty} d^{p m}\left(x_{n}, x_{n+1}\right)=d^{*} \geq 0$. Suppose that $d^{*}>0$.

By the other hand (12) became

$$
\tau+F\left(d^{p m}\left(x_{n}, x_{n+1}\right)\right) \leq F\left(\mu \cdot d^{p m}\left(x_{n-1}, x_{n}\right)+(1-\mu) \cdot d^{p m}\left(x_{n}, x_{n+1}\right)\right),
$$

where $\mu=\alpha+\beta+\delta+L$. Since $F$ is strictly increasing there exists $\lim _{\tilde{\zeta} \rightarrow d^{*}} F(\xi)=F\left(d^{*}+\right)$, so taking the limit as $n \rightarrow+\infty$ in (18) we get $\tau+F\left(d^{*}+\right) \leq F\left(d^{*}+\right)$, which is a contradiction. Hence, $\lim _{n \rightarrow+\infty} d^{p m}\left(x_{n}, x_{n+1}\right)=0$. 
Now, we can show that $\left\{x_{n}\right\}_{n \in \mathbb{N} \cup\{0\}}$ is a $0-d^{p m}$-Cauchy sequence. If it is not, putting in (12) $x=x_{p(k)}, y=x_{n(k)}$ we get:

$$
\tau+F\left(d^{p m}\left(x_{p(k)+1}, x_{n(k)+1}\right)\right) \leq F\left(A\left(x_{p(k)}, x_{n(k)}\right)\right),
$$

where $A\left(x_{p(k)}, x_{n(k)}\right)=\alpha \cdot d^{p m}\left(x_{p(k)}, x_{n(k)}\right)+\beta \cdot d^{p m}\left(x_{p(k)}, x_{p(k)+1}\right)$

$+\gamma \cdot d^{p m}\left(x_{n(k)}, x_{n(k)+1}\right)+\delta \cdot d^{p m}\left(x_{p(k)}, x_{n(k)+1}\right)+L \cdot d^{p m}\left(x_{p(k)+1}, x_{n(k)}\right)$. Further, according to Proposition 1. (vi) we get $\lim _{k \rightarrow+\infty} A\left(x_{p(k)}, x_{n(k)}\right)=(\alpha+0+0+\delta+L) \varepsilon^{+} \leq$ $(\alpha+\delta+2 L) \varepsilon^{+}$. Since $\alpha+\beta+\gamma+2 \delta+L=1$, then $\alpha+\delta+L \leq 1, \operatorname{solim}_{k \rightarrow+\infty} A\left(x_{p(k)}, x_{n(k)}\right)$ $\leq \varepsilon^{+}$. Now, taking the limit in (19) as $k \rightarrow+\infty$ we obtain $\tau+F\left(\varepsilon^{+}+\right)$ $\leq F\left((\alpha+\delta+2 L) \varepsilon^{+}+\right) \leq F\left(\varepsilon^{+}+\right)$, which is a contradiction. Hence, we have proved that $\left\{x_{n}\right\}_{n \in \mathbb{N} \cup\{0\}}$ is a $0-d^{p m}$-Cauchy sequence. Since $\left(X, d^{p m}\right)$ is a $0-d^{p m}$-complete partial metric space, then $\left\{x_{n}\right\}_{n \in \mathbb{N} \cup\{0\}}$ converges to some point $\bar{x}$ in X. By (17) and Proposition 1. (vii) it follows that $\bar{x}, T \bar{x} \notin\left\{x_{n}\right\}_{n \geq n_{1}}$ for some $n_{1} \in \mathbb{N}$. Assume that $T \bar{x} \neq \bar{x}$. Then according to (13) we get

$$
\begin{gathered}
d^{p m}(\bar{x}, T \bar{x}) \leq d^{p m}\left(\bar{x}, x_{n+1}\right)+d^{p m}\left(T x_{n}, T \bar{x}\right) \\
<d^{p m}\left(\bar{x}, x_{n+1}\right)+\alpha \cdot d^{p m}\left(x_{n}, \bar{x}\right)+\beta \cdot d^{p m}\left(x_{n}, x_{n+1}\right)+\gamma \cdot d^{p m}(\bar{x}, T \bar{x}) \\
+\delta \cdot d^{p m}\left(x_{n}, T \bar{x}\right)+L \cdot d^{p m}\left(T x_{n}, \bar{x}\right),
\end{gathered}
$$

for $n \geq n_{1}$. Since, $d^{p m}\left(x_{n}, T \bar{x}\right) \leq d^{p m}\left(x_{n}, \bar{x}\right)+d^{p m}(\bar{x}, T \bar{x})-d^{p m}(\bar{x}, \bar{x})=d^{p m}\left(x_{n}, \bar{x}\right)+$ $d^{p m}(\bar{x}, T \bar{x})-0=d^{p m}\left(x_{n}, \bar{x}\right)+d^{p m}(\bar{x}, T \bar{x})$ we further have that

$$
\begin{gathered}
d^{p m}(\bar{x}, T \bar{x}) \leq d^{p m}\left(\bar{x}, x_{n+1}\right)+\alpha \cdot d^{p m}\left(x_{n}, \bar{x}\right)+\beta \cdot d^{p m}\left(x_{n}, x_{n+1}\right)+\gamma \cdot d^{p m}(\bar{x}, T \bar{x}) \\
+\delta \cdot d^{p m}\left(x_{n}, \bar{x}\right)+\delta \cdot d^{p m}(\bar{x}, T \bar{x})+L \cdot d^{p m}\left(x_{n+1}, \bar{x}\right) \\
\rightarrow 0+\alpha \cdot 0+\beta \cdot 0+(\gamma+\delta) \cdot d^{p m}(\bar{x}, T \bar{x})+\delta \cdot 0+L \cdot 0=(\gamma+\delta) \cdot d^{p m}(\bar{x}, T \bar{x}) \\
<1 \cdot d^{p m}(\bar{x}, T \bar{x}),
\end{gathered}
$$

which is a contradiction. Hence, $\bar{x}$ is a unique fixed point of $T$.

It is worth to noticing that from our Theorem 4 follow several significant results in the context of 0-complete partial metric spaces. As a first we have the following:

Corollary 2. Let $\left(X, d^{p m}\right)$ be a $0-d^{p m}$-complete partial metric space and $T$ be a self-mapping on $X$. Assume that there exist $F:(0,+\infty) \rightarrow(-\infty,+\infty)$ a strictly increasing mapping and $\tau>0$ such that

$$
\tau+F\left(d^{p m}(T x, T y)\right) \leq F\left(d^{p m}(x, y)\right),
$$

for all $x, y \in X$ with $d^{p m}(T x, T y)>0$. Then $T$ has a unique fixed point $\bar{x}$ in $X$ and for every $x \in X$ the sequence $\left\{T^{n} x\right\}_{n \in \mathbb{N} \cup\{0\}}$ converges to $\bar{x}$.

Proof. Taking $\alpha=1, \beta=\gamma=\delta=L=0$ in Theorem 4 the result follows.

Also taking in Theorem $4, \delta=L=0$ (resp. $\alpha=\delta=L=0 ; \alpha=\beta=\gamma=0$ ) we get Reich (resp. Kannan; Chatterjea) type theorem in the context of $0-d^{p m}$-complete partial metric spaces where $F:(0,+\infty) \rightarrow(-\infty,+\infty)$ is a strictly increasing mapping.

The following new result shows that the monotonicity condition of $F$ is not necessary:

Theorem 5. Let $T$ be a self-mapping of a $0-d^{p m}$-complete partial metric space $\left(X, d^{p m}\right)$. Suppose there exists $\tau>0$ such that for all $x, y \in X, d^{p m}(T x, T y)>0$ yields

$$
\tau+F\left(d^{p m}(T x, T y)\right) \leq F\left(d^{p m}(x, y)\right),
$$


where $F:(0,+\infty) \rightarrow(-\infty,+\infty)$ is a mapping satisfying the conditions (F2) and (F3").

Then $T$ has a unique fixed point $x^{*} \in X$ and for every $x \in X$ the sequence $\left\{T^{n} x\right\}_{n \in \mathbb{N} \cup\{0\}}$ converges to $x^{*}$.

Proof. First, if $T$ has a fixed point then (23) yields that it is a unique. Further, if $x_{0}$ is an arbitrary point in $X$ and the sequence $x_{n}=T x_{n-1}, n \in \mathbb{N}$ is the corresponding Picard's sequence with $x_{p}=x_{p-1}$ for some $p \in \mathbb{N}$ then $x_{p-1}$ is a unique fixed point of $T$. Therefore, let $x_{n} \neq x_{n-1}$ for all $n \in \mathbb{N}$. Hence, we assume that $0<d^{p m}\left(x_{n-1}, T x_{n-1}\right)=d^{p m}\left(x_{n-1}, x_{n}\right)$ for all $n \in \mathbb{N}$. Now, by the hypothesis we get

$$
F\left(d^{p m}\left(x_{n}, x_{n+1}\right)\right) \leq \ldots \leq F\left(d^{p m}\left(x_{0}, x_{1}\right)\right)-n \tau \rightarrow-\infty,
$$

as $n \rightarrow+\infty$. Hence, according to (F2) it follows that $d^{p m}\left(x_{n}, x_{n+1}\right) \rightarrow 0$ as $n \rightarrow+\infty$. Further it is clear that $F \circ d^{p m}\left(x_{n}, x_{n+1}\right)<F \circ d^{p m}\left(x_{n-1}, x_{n}\right)$ from which it follows $x_{n} \neq x_{m}$ if $n \neq m$. Now we can claim that $\left\{x_{n}\right\}_{n \in \mathbb{N}}$ is a $0-d^{p m}$-Cauchy. Indeed, if it is not, putting $x=x_{p(k)}, y=x_{n(k)}$ in (23) we obtain

$$
\tau+F\left(d^{p m}\left(x_{p(k)+1}, x_{n(k)+1}\right)\right) \leq F\left(d^{p m}\left(x_{p(k)}, x_{n(k)}\right)\right) .
$$

By (F3"), taking the limit in (25) as $k \rightarrow+\infty$, we get $\tau+F(\varepsilon+) \leq F(\varepsilon+)$, which is a contradiction. Therefore, $\left\{x_{n}\right\}_{n \in \mathbb{N}}$ is a $0-d^{p m}$-Cauchy sequence. Since, $\left(X, d^{p m}\right)$ is a $0-d^{p m}$-complete it yields that $\left\{x_{n}\right\}_{n \in \mathbb{N}}$ converges to some point $\bar{x} \in X$. We shall prove that $T \bar{x}=\bar{x}$. If it is not true, then (because $x_{n} \neq x_{m}$ if $n \neq m$ ) there exists $n_{1} \in \mathbb{N}$ such that $\bar{x}, T \bar{x} \notin\left\{x_{n}\right\}_{n \geq n_{1}}$. Further, for such $n$ we have

$$
\tau+F\left(d^{p m}\left(x_{n+1}, T \bar{x}\right)\right) \leq F\left(d^{p m}\left(x_{n}, \bar{x}\right)\right) .
$$

By (F3"), taking the limit as $k \rightarrow+\infty$ in (26) we get $\lim _{n \rightarrow+\infty} F\left(d^{p m}\left(x_{n+1}, T \bar{x}\right)\right)=$ $-\infty=F\left(\lim _{n \rightarrow+\infty} d^{p m}\left(x_{n+1}, T \bar{x}\right)\right)$. Now, by (F2) and Proposition 1. (i) yields that $\lim _{n \rightarrow+\infty} d^{p m}\left(x_{n+1}, T \bar{x}\right)=0=d^{p m}(\bar{x}, T \bar{x})$. This is a contradiction. Therefore, $\bar{x}$ is a fixed point of $T$. Theorem is completely proved.

Our third new result here is the extension of Theorems 2 and 3 from the ordinary metric space to metric-like space. The proofs are very similar to the proofs for Theorems 4 and 5 and that is why we omit them.

Theorem 6. Let $T$ be a self-mapping of a $0-d^{m l}$-complete metric-like space $\left(X, d^{m l}\right)$. Suppose there exists $\tau>0$ such that for all $x, y \in X, d^{m l}(T x, T y)>0$ yields

$$
\tau+F\left(d^{m l}(T x, T y)\right) \leq F(A(x, y))
$$

where $F:(0,+\infty) \rightarrow(-\infty,+\infty)$ is a strictly increasing mapping, $A(x, y)=\alpha \cdot d^{m l}(x, y)+$ $\beta \cdot d^{m l}(x, T x)+\gamma \cdot d^{m l}(y, T y)+\delta \cdot d^{m l}(x, T y)+L \cdot d^{m l}(y, T x), \alpha, \beta, \gamma, \delta, L$ are non-negative numbers $\delta<\frac{1}{2}, \gamma<1, \alpha+\beta+\gamma+2 \delta+2 L=1,0<\alpha+\delta+L \leq 1$. Then $T$ has a unique fixed point $x^{*} \in X$ and for every $x \in X$ the sequence $\left\{T^{n} x\right\}_{n \in \mathbb{N} \cup\{0\}}$ converges to $x^{*}$.

In the following result as in Theorem 5 we show that the monotonicity condition of $F$ is not necessary:

Theorem 7. Let $T$ be a self-mapping of a $0-d^{m l}$-complete metric-like space $\left(X, d^{m l}\right)$. Assume that there exists $\tau>0$, such that for all $\bar{x}, \bar{y} \in X, d^{m l}(T \bar{x}, T \bar{y})>0$ yields

$$
\tau+F\left(d^{m l}(T \bar{x}, T \bar{y})\right) \leq F\left(d^{m l}(\bar{x}, \bar{y})\right),
$$


where $F:(0,+\infty) \rightarrow(-\infty,+\infty)$ is a mapping satisfying the conditions (F2) and (F3"), where $\left(F 3^{\prime \prime}\right): F$ is continuous on $(0, \alpha)$, with $\alpha$ a positive real number.

Then, the mapping $T$ has a unique fixed point $\bar{x} \in X$ and for every $x \in X$ the sequence $\left\{T^{n} x\right\}_{n \in \mathbb{N} \cup\{0\}}$ converges to $\bar{x}$.

As the immediate corollaries of Theorem 4 we obtain several new contractive conditions that supplement the ones given in $[19,29]$.

Corollary 3. Let $T$ be a self-mapping of a $0-d^{p m}$-complete partial metric space $\left(X, d^{p m}\right)$. Suppose there exist $\tau_{i}>0, i=\overline{1,9}$ such that for all $x, y \in X, d^{p m}(T x, T y)>0$ it follows

$$
\begin{aligned}
& \tau_{1}+d^{p m}(T x, T y) \leq A(x, y), \\
& \tau_{2}+\exp \left(d^{p m}(T x, T y)\right) \leq \exp (A(x, y)) \\
& \tau_{3}-\frac{1}{d^{p m}(T x, T y)} \leq-\frac{1}{A(x, y)}, \\
& \tau_{4}-\frac{1}{d^{p m}(T x, T y)}+d^{p m}(T x, T y) \leq-\frac{1}{A(x, y)}+A(x, y), \\
& \tau_{5}+\frac{1}{1-\exp \left(\left(d^{p m}(T x, T y)\right)\right.} \leq \frac{1}{1-\exp (A(x, y))}, \\
& \tau_{6}+\frac{1}{\exp \left(-d^{p m}(T x, T y)\right)-\exp \left(d^{p m}(T x, T y)\right)} \leq \frac{\exp (-A(x, y))-\exp (A(x, y))}{\tau_{7}+\left(d^{p m}(T x, T y)\right)^{s} \leq(A(x, y))^{s}, s>0,}
\end{aligned}
$$

where $A(x, y)=\alpha \cdot d^{p m}(x, y)+\beta \cdot d^{p m}(x, T x)+\gamma \cdot d^{p m}(y, T y)+\delta \cdot d^{p m}(x, T y)+L \cdot d^{p m}(y, T x)$ while $\alpha, \beta, \gamma, \delta, L$ are non-negative numbers: $\delta<\frac{1}{2}, \gamma<1, \alpha+\beta+\gamma+2 \delta+L=1$, $0<\alpha+\delta+L \leq 1$. Then in every of these cases (29)-(35) Thas a unique fixed point $\bar{x} \in X$ and for $x \in X$ the sequence $\left\{T^{n} x\right\}_{n \in \mathbb{N} \cup\{0\}}$ converges to $\bar{x}$.

Proof. Take in Theorem $4, F(\eta)=\eta, F(\eta)=\exp \eta, F(\eta)=-\frac{1}{\eta}, F(\eta)=-\frac{1}{\eta}+\eta, F(\eta)=$ $\frac{1}{1-\exp \eta}, F(\eta)=\frac{1}{\exp (-\eta)-\exp \eta}, F(\eta)=\eta^{s}$ respectively. Because each of the mappings $\eta \mapsto F(\eta)$ is strictly increasing on $(0,+\infty)$ the result is an immediate consequence of Theorem 4 .

Finally, we state an application of Theorem 6 for solving fractional differential equations. This is in fact a support for our theoretical result established in Theorem 6. We will use metric like distance $d^{m l}$.

For $f:[0,+\infty) \rightarrow \mathbb{R}$ a continuous function we recall the Caputo derivative of function $f$ order $\alpha>0$ as follows, see $[30,31]$

$$
{ }^{C} D^{\alpha}(f(t)):=\frac{1}{\Gamma(n-\alpha)} \int_{0}^{t}(t-s)^{n-\alpha-1} f^{(n)}(s) d s(n-1<\alpha<n, n=[\alpha]+1),
$$

where $[\alpha]$ denotes the integer part of the positive real number $\alpha$ and $\Gamma$ is a well known gamma function.

Our main purpose is to give an application to Theorem 6 to prove the existence of the solution for nonlinear fractional differential equation

$$
{ }^{C} D^{\alpha}(x(t))+g(t, x(t))=0(0 \leq t \leq 1, \alpha<1)
$$

with the boundary conditions $x(0)=0=x(1)$, where $x \in C([0,1], \mathbb{R})$ and $C([0,1], \mathbb{R})$ is the set of all continuous functions from $[0,1]$ to $\mathbb{R}$ and $g:[0,1] \times \mathbb{R} \rightarrow \mathbb{R}$ is a continuous 
function, see [32]. Let us give the Green's function associated with the problem (36) as follows

$$
G(t, s)=\left\{\begin{array}{l}
(t(1-s))^{\beta-1}-(t-s)^{\beta-1}, \text { if } 0 \leq s \leq t \leq 1 \\
\frac{(t(1-s))^{\beta-1}}{\Gamma(\beta)}, \text { if } 0 \leq t \leq s \leq 1 .
\end{array}\right.
$$

Now we give the next main result that support our Theorem 6 .

Theorem 8. Consider the nonlinear fractional differential equation (36). Let $\varkappa: \mathbb{R} \times \mathbb{R} \rightarrow \mathbb{R}$ be a given mapping and $g:[0,1] \times \mathbb{R} \rightarrow \mathbb{R}$ be a continuous function. Suppose that the following assertions are true:

(i) there exists $x_{0} \in C([0,1], \mathbb{R})$ such that $\varkappa\left(x_{0}(t), \int_{0}^{t} T x_{0}(t)\right) \geq 0$ for all $t \in[0,1]$, where $T: C([0,1], \mathbb{R}) \rightarrow C([0,1], \mathbb{R})$ is defined by

$$
T x(t)=\int_{0}^{1} G(t, s) g(s, x(s)) d s ;
$$

(ii) there exists $\tau>0$ such that for all $x, y \in X, d^{m l}(T x, T y)>0$ and $d^{m l}(x, y)>0$ yields

$$
|g(t, a)|+|g(t, b)| \leq A(x, y) e^{-\tau},
$$

for all $t \in[0,1]$ and $a, b \in \mathbb{R}$ with $\varkappa(a, b) \geq 0$ where $A(x, y)=\alpha \cdot d^{m l}(x, y)+\beta$. $d^{m l}(x, T x)+\gamma \cdot d^{m l}(y, T y)+\delta \cdot d^{m l}(x, T y)+L \cdot d^{m l}(y, T x), \alpha, \beta, \gamma, \delta, L$ are non-negative numbers, $\delta<\frac{1}{2}, \gamma<1, \alpha+\beta+\gamma+2 \delta+2 L=1,0<\alpha+\delta+L \leq 1$.

(iii) for each $t \in[0,1]$ and $x, y \in C([0,1], \mathbb{R}), \varkappa(x(t), y(t)$ implies $x(T x(t), T y(t))) \geq 0$;

(iv) for each $t \in[0,1]$, if $\left\{x_{n}\right\}$ is a sequence in $C([0,1], \mathbb{R})$ such that $x_{n} \rightarrow x^{*}$ in $C([0,1], \mathbb{R})$ and $\varkappa\left(x_{n}(t), x_{n+1}(t)\right) \geq 0$ for all $n \in \mathbb{N}$, then $\varkappa\left(x_{n}(t), x(t)\right) \geq 0$ for all $n \in \mathbb{N}$.

Then problem (36) has at least one solution.

Proof. Let $\left(X, d^{m l}\right)$ endowed with the metric-like

$$
\left.d^{m l}(x, y)=\sup _{t \in[0,1]}(|x(t)|+|y(t)|), \text { for all } x, y \in X\right) .
$$

We can prove easily that $\left(X, d^{m l}\right)$ is a 0-complete metric-like space.

Obviously $x^{*} \in X$ is a solution of (36) if and only if $x^{*} \in X$ is a solution of the equation $x(t)=\int_{0}^{1} G(t, s) g(s, x(s)) d s$ for all $t \in[0,1]$. Then problem (36) can be considered to find an $x^{*} \in X$ which is a fixed point for the mapping $T$.

Let $x, y \in X$ such that $\varkappa(x(t), y(t)) \geq 0$ for all $t \in[0,1]$. By (iii) we have $\varkappa(T x, T y)>0$. Then, by (i) and (ii) we get the next inequalities

$$
\begin{gathered}
|T x(t)|+|T y(t)|=\left|\int_{0}^{1} G(t, s) g(s, x(s)) d s\right|+\left|\int_{0}^{1} G(t, s) g(s, y(s)) d s\right| \\
\leq \sup _{t \in[0,1]}(|g(s, x(s))|+|g(s, y(s))|) \sup _{t \in[0,1]}\left|\int_{0}^{1} G(t, s) d s\right| \\
\leq \sup _{t \in[0,1]} A(x, y) e^{-\tau} .
\end{gathered}
$$


This means

$$
d^{m l}(T x, T y) \leq A(x, y) e^{-\tau}
$$

If we take $F(r)=\ln (r)$ for $r>0$ and since $F$ is strictly increasing we get

$$
\ln \left(d^{m l}(T x, T y)\right) \leq \ln \left(A(x, y) e^{-\tau}\right)=\ln (A(x, y))-\tau .
$$

Equivalently

$$
\tau+F\left(d^{m l}(T x, T y)\right) \leq F(A(x, y))
$$

where $F:(0,+\infty) \rightarrow(-\infty,+\infty)$ is a strictly increasing mapping, $A(x, y)=\alpha \cdot d^{m l}(x, y)+$ $\beta \cdot d^{m l}(x, T x)+\gamma \cdot d^{m l}(y, T y)+\delta \cdot d^{m l}(x, T y)+L \cdot d^{m l}(y, T x), \alpha, \beta, \gamma, \delta, L$ are non-negative numbers, $\delta<\frac{1}{2}, \gamma<1, \alpha+\beta+\gamma+2 \delta+2 L=1,0<\alpha+\delta+L \leq 1$.

Applying Theorem 6. we deduce that $T$ has a fixed point, which yields that the Equation (36) has at least one solution.

\section{Conclusions}

It can be checked that each $d^{m}$-complete metric space $\left(X, d^{m}\right)$ is a $0-d^{p m}$-complete partial metric space and each $0-d^{p m}$ - complete partial metric space is a $0-d^{m l}$-complete metric-like space. The opposite in both cases is not true. Namely, there exists $0-$ $d^{m l}$-complete metric-like space that is not $0-d^{p m}$-complete partial metric space, i.e., there exists $0-d^{p m}$-complete partial metric space that is not $d^{m}$-complete metric space. The main result in this manuscript is among other things, the proper generalization of Theorem 2.1 in [21] established for $d^{m}$-complete metric spaces to 0 - $d^{p m}$-complete partial metric spaces, that is, to $0-d^{m l}-$ complete metric like spaces. For the proof of our main result we have used only the property (F1) from Definition 5.

Author Contributions: Conceptualization S.R.; methodology, N.M. and S.R.; formal analysis, N.M. and L.R.P.; writing-original draft preparation, N.M. and L.R.P.; supervision, S.R. All authors have read and agreed to the published version of the manuscript.

Funding: This research received no external funding.

Data Availability Statement: Not applicable.

Acknowledgments: The research of authors N.M. and L.R.P. was funded by the Ministry of Education, Science and Technological Development of the Republic of Serbia.

Conflicts of Interest: The authors declare no conflict of interest.

\section{References}

1. Fréchet, M. Sur quelques points du calcul functionnel. Rendic. Circ. Mat. Palermo 1906, 22, 1-74. [CrossRef]

2. Todorčević, V. Harmonic Quasiconformal Mappings and Hyperbolic Type Metrics; Springer: Cham, Switzerland, 2019.

3. Matthews, S.G. Partial metric topology. Ann. N. Y. Acad. Sci. Pap. Ed. 1994, 728, 183-197. [CrossRef]

4. Nashine, H.K.; Kadelburg, Z.; Radenović, S.; Kim, J.K. Fixed point theorems under Hardy-Rogers contractive conditions on 0-complete ordered partial metric spaces. Fixed Point Theory Appl. 2012, 2012, 180. [CrossRef]

5. Radenović, S. Classical fixed point results in 0-complete partial metric spaces via cyclic-type extension. Allahabad Math. Soc. 2016, 31, 39-55.

6. Rajić, V.Ć.; Radenović, S.; Chauhan, S. Common fixed point of generalized weakly contractive maps in partial metric spaces. Acta Math. Sci. 2014, 34, 1345-1356. [CrossRef]

7. Shukla, S.; Radenović, S.; Rajić, V.Ć. Some common fixed point theorems in 0- $\sigma$-complete metric-like spaces. Vietnam J. Math. 2013, 41, 341-352. [CrossRef]

8. Shukla, S.; Radenović, S.; Kadelburg, Z. Some fixed point theorems for ordered F-generalized contractions in o-f-orbitally complete partial metric spaces. Theory Appl. Math. Comput. Sci. 2014, 4, 87-98.

9. Vujaković, J.; Mitrović, S.; Mitrović, Z.D.; Radenović, S. On F-contractions for weak $\alpha$-admissible mappings in metric-like spaces. Mathematics 2020, 8, 1629. [CrossRef]

10. Amini-Harandi, A. Metric-like spaces, partial metric spaces and fixed points. Fixed Point Theory Appl. 2012, 2012, 204. [CrossRef]

11. Joshi, V.; Singh, D.; Singh, A. Applicative approach of fixed point theorems towards various engineering problems. Fixed Point Theory 2020, 21, 595-610. [CrossRef] 
12. Karapinar, E.; Kutbi, M.A.; Piri, H.; O'Regan, D. Fixed point of conditionally F-contraction in complete metric-like spaces. Fixed Point Theory Appl. 2015, 2015, 126. [CrossRef]

13. Salimi, P.; Hussain, N.; Shukla, S.; Fathollahi, S.; Radenović, S. Fixed point results for cyclic $\alpha-\psi \phi$-contractions with applications to integral equations. J. Comput. Appl. Math. 2015, 290, 445-458. [CrossRef]

14. Abbas, M.; Jungck, G. Common fixed point results for noncommuting mappings without continuity in cone metric space. J. Math Anal. Appl. 2008, 341, 416-420. [CrossRef]

15. Banach, S. Sur les opérations dans les ensembles abstraits et leur application aux équations intégrales. Fundam. Math. 1922, 3, 133-181. [CrossRef]

16. Fabiano, N.; Parvaneh, V.; Mirković, D.; Paunović, L.; Radenović, S. On W-contractions of Jungck-Ćirić-Wardowski-type in metric spaces. Cogent Math. Statist. 2020, 7, 1792699. [CrossRef]

17. George, R.; Mitrović, A.D.; Radenović, S. On some coupled fixed points of generalized T-contraction mapping in a $\mathrm{b}_{v}(s)$-metric spaces and its application. Axioms 2020, 9, 129. [CrossRef]

18. Piri, H.; Kumam, P. Some fixed point theorems concerning F-contraction in complete metric spaces. Fixed Point Theory Appl. 2014, 2014, 210. [CrossRef]

19. Rhoades, B.E. A comparison of various definitions of contractive mappings. Trans. Amer. Math. Soc. 1997, 226, 257-290. [CrossRef]

20. Secelean, N.A. Iterated function system consisting of F-contractions. Fixed Point Theory Appl. 2013, 2013, 277. [CrossRef]

21. Wardowski, D. Fixed points of a new type of contractive mappings in complete metric spaces. Fixed Point Theory Appl. 2012, 2012, 94. [CrossRef]

22. Singh, D.; Joshi, V.; Imdad, M.; Kumam, P. Fixed point theorems via generalized F-contraction with applications to functional equations accuring in dynamic programing. J. Fixed Point Theory Appl. 2017, 19, 1453-1479. [CrossRef]

23. Carić, B.; Došenović, T.; George, R.; Mitrović, Z.D.; Radenović, S. On Jungck-Branciari-Wardowski type fixed point results. Mathematics 2021, 9, 161. [CrossRef]

24. Popescu, O.; Stan, G. Two fixed point theorems concerning F-contraction in complete metric spaces. Symmetry 2020, $12,58$. [CrossRef]

25. Vujaković, J.; Ljajko, E.; Radojević, S.; Radenović, S. On some new Jungck-Fisher-Wardowski type fixed point results. Symmetry 2020, 12, 2048. [CrossRef]

26. Vujakov ić, J.; Radenović, S. On Some F-Contraction of Piri-Kumam-Dung-Type Mappings in Metric Spaces. Vojn. Tehnički Glas./Mil. Tech. Cour. 2020, 68, 697-714.

27. Vujakovi ć, J.; Ljajko, E.; Pavlović, M.; Radenović, S. On some new contractive conditions in complete metric spaces. Mathematics 2021, 1, 9000. [CrossRef]

28. Consentino, V.; Vetro, P. Fixed point results for F-contractive mappings of Hardy-Rogers-type. Filomat 2014, $28,715-722$. [CrossRef]

29. Collaco, P.; Silva, J.C. A complete comparison of 23 contraction conditions. Nonlinear Anal. Theory Methods Appl. 1997, 30, 471-476. [CrossRef]

30. Kilb, A.A.; Srivastava, H.H.; Trujillo, J.J. Theory and Applications of Fractional Differential Equations; North-Holand Mathematics Studies; Elesevier: Amsterdam, The Netherlands, 2006; Volume 204.

31. Samko, S.G.; Kilbas, A.A.; Marichev, O.I. Fractional Integral and Derivative; Gordon and Breach: London, UK, 1993.

32. Podlubni, I. Fractional Differential Equations; Academic Press: San Diego, CA, USA, 1999. 